\title{
INTERGENERATIONAL TRANSMISSION OF PERCEIVED PARENTAL REARING STYLES: A THREE GENERATION FAMILIES STUDY
}

\author{
F. Lopes, $\boldsymbol{H}$. Espirito-Santo, H. Vicente
}

Psychology, Instituto Superior Miguel Torga, Coimbra, Portugal

Introduction: The transmission of perceived parental rearing styles through generations has been proved in several studies, mostly in studies with two generations samples.

Objectives/aims: The main aim of this study is to investigate the intergenerational transmission of the perception of parental rearing styles in families composed by three generations.

Methodology: A convenience sample of 143 participants was collected, belonging to a female lineage subsystem, divided in three generations (G1/grandmother, $n=41$, mean age $=74.8 ; S D=6.26$; $\mathrm{G} 2 /$ mother, $n=41$; mean age $=42.4 ; S D=5.61$; $\mathrm{G} 3 /$ daughter, $n=41$ mean age $=22.3 ; S D=4.50$ ). All the participants completed a questionnaire regarding sociodemographic, family and educational aspects, and the EMBU Inventory - My Memories of Upbringing.

Results: Spearman correlations revealed several significant associations on the EMBU Inventory dimensions between $\mathrm{G} 1$ and G2. The G1 experiences of Emotional Warmth, Overprotection and Rejection, regarding mother and father, are correlated with the $\mathrm{G} 2$ experiences concerning the same dimensions. No significant associations were found in the same dimensions of the instrument between $\mathrm{G} 2$ and $\mathrm{G} 3$.

Discussion/conclusion: The results suggest the intergenerational transmission of the perception of parental rearing styles from $G 1$ to $G 2$, but not from $G 2$ to $G 3$. These findings provide partial support to the intergenerational transmission hypothesis, adding to the body of knowledge about the transmission of parenting styles across generations and the multigenerational family in which it occurs. Further studies are needed to elucidate the apparent absence of intergenerational transmission between $\mathrm{G} 2$ and $\mathrm{G} 3$. 